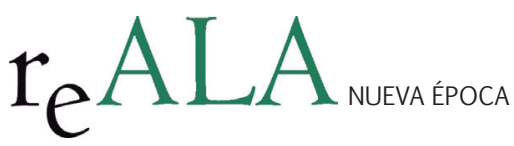

REALA, n Extraordinario, enero 2015 ISSN: 1989-8975

DOI: http://dx.doi.org/10.24965/reala.voiExtra.10221

\title{
Oportunidades, riesgos y resultados reales de la Ley de racionalización y sostenibilidad de la Administración Local'
}

\author{
Ricardo Rivero Ortega \\ Catedrático de Derecho Administrativo. Universidad de Salamanca \\ ricriv@usal.es
}

\section{Resumen}

Un año después de la entrada en vigor de la Ley de Racionalización y Sostenibilidad de la Administración local podemos valorar su necesidad, oportunidades y riesgos creados. La aprobación de la norma en un momento de crisis económica explica algunas de sus soluciones, que sin embargo no pueden considerarse radicales. La efectividad de algunas de sus previsiones se enfrenta ahora a las medidas adoptadas por los poderes autonómicos, en una actitud defensiva de sus propias competencias y de los servicios municipales afectados.

Palabras clave

Reforma local. Medidas de ajuste. Competencias del Estado y de las Comunidades Autónomas sobre la Administración local. Servicios públicos.

\section{Opportunities, Risks, and Actual Results and Sustainability Rationalization Act Local Government}

\section{Abstract}

One year after the entry into force of the Act of rationalization and sustainability of the Local Administration we can value its necessity, opportunities and risks created. The adoption of the law in a time of economic crisis explains some of its solutions, which however cannot be considered radicals. The effectiveness of some of its provisions is now faced with the measures taken by autonomous governments, in a defensive attitude to their own competences and the municipal services concerned.

Key words

Local reform. Adjustment measures. Competences of State and of the Autonomous Communities on Local Government. Public services.

$1 \quad$ El presente trabajo se ha elaborado en el marco del Proyecto de investigación, “El Derecho de la Administración sostenible: mejores normas para la austeridad, la eficiencia y la rendición de cuentas”, (DER 2011-228014), financiado por el Ministerio de Economía y Competitividad. 


\title{
SUMARIO
}

\begin{abstract}
1. ¿ERA NECESARIA ESTA REFORMA DEL RÉGIMEN LOCAL EN ESPAÑA?. 2. OPORTUNIDADES DE LA LEY DE RACIONALIZACIÓN. 3. RIESGOS DE LA LEY DE RACIONALIZACIÓN Y SOSTENIBILIDAD. 4. LA DESACTIVACIÓN DE LA LEY POR LOS PODERES AUTONÓMICOS. 5. PERSPECTIVAS DE FUTURO.
\end{abstract}

\section{1. ¿ERA NECESARIA ESTA REFORMA DEL RÉGIMEN LOCAL EN ESPAÑA?}

A un año de la publicación en el BOE de la Ley 27/2013, de 27 de diciembre, de Racionalización y Sostenibilidad de la Administración local, podemos ponderar con suficiente perspectiva el grado de oportunidad y la contribución efectiva de la reforma a la mejora de la Administración y el Gobierno local: ¿Se encuentran municipios y diputaciones hoy en mejores condiciones de afrontar sus desafíos? ¿Han innovado realmente en la manera de prestar los servicios públicos hacia los ciudadanos?²

Cuando nos detenemos a analizar las estrategias adoptadas por los entes locales para responder a la crisis económica, nos encontramos con ajustes y recortes lineales en sus gastos de personal; también con la restricción del alcance de determinados servicios. Vemos muchas menos iniciativas en clave de sostenibilidad, sin reducir personal ni prestaciones. Casi todas las decisiones adoptadas antecedieron además a la aprobación de la nueva Ley, propiciando el ajuste otras medidas: ¿hacían falta alforjas para este viaje?33

Más allá, ¿era necesario tanto estrépito? Recordemos la enorme polémica suscitada en torno a la tramitación y promulgación de esta Ley, proyectada especialmente sobre algunos de sus contenidos principales. No se discutía toda ella, como tampoco se ha cuestionado en los últimos años la necesidad de una reforma de la Ley de Bases de Régimen Local en España. Se presentaba abierta oposición contra su trayectoria escorada, no se discutía la inadaptación de numerosas previsiones del régimen anterior al contexto actual ${ }^{4}$.

De hecho, en pasadas legislaturas se abrieron procesos de elaboración de borradores de anteproyecto de modificación de la Ley de Bases, pudiendo recordarse el Libro Blanco para la reforma del gobierno local en España, un documento promovido en 2005 por el Ministerio de Administraciones públicas a través de su Secretaría de Estado para de cooperación territorial. Algunos de los temas tratados en este documento coinciden con puntos clave de la reforma actual: competencias, estructuras, financiación 5 .

Desde posiciones oficiales identificadas con el texto de los sucesivos borradores de anteproyecto se sostienen razones verosímiles para justificar su enfoque, derivado de la reforma constitucional del artículo 135, tan cuestionada ${ }^{6}$. Una defensa de la constitucionalidad del texto resulta mucho más sólida desde la tesis del cambio en nuestra Constitución económica resultante de la nueva cláusula, Ilamada a producir efectos múltiples, competenciales y organizativos?

Y es que la crisis económica está en el origen de esta reforma, incluida en el paquete de compromisos asumidos por el Gobierno de España con la Unión Europea en un contexto de riesgo de quiebra. Si otros países intervenidos - como Portugal o Grecia - tuvieron que reducir drásticamente sus estructuras locales, el nuestro evitó tan draconianas exigencias, pero hubo de cuantificar incrementos de eficiencia en este ámbito. Al fin había que poner cifras sobre la mesa para recibir el visto bueno ${ }^{8}$.

A nadie se le escapa que las circunstancias lo exigían, pero el cálculo de los 8000 millones de euros estimados como ahorro derivado de esta reforma fue objeto de todo tipo de críticas, y no sólo por lo incierto de su fundamento y sus efectos sobre los presupuestos y servicios municipales. No cabe sin embargo tildarlo de exagerado, pues la contribución de las entidades locales a la reducción del déficit público, antes incluso de entrar en vigor la reforma, estuvo en el entorno de tal cifra'.

2 Vid. RIVERO ORTEGA, Ricardo/MERINO ESTRADA, Valentín, Innovación y gobiernos locales. Estrategias innovadoras de municipios y diputaciones en un contexto de crisis, INAP, Madrid, 2014

3 Vid. RIVERO ORTEGA, Ricardo, “Algunas claves para la elaboración de un Plan de Ajuste Municipal”, Diario de Derecho municipal, lustel, marzo 2012.

4 Vid. RIVERO ORTEGA, Ricardo/MERINO ESTRADA, Valentín, “La reforma del régimen local en España: necesidad, alcance, orientación y contenidos básicos", Revista de Estudios Locales, Recopilatorio 2013.

5 Vid. Libro Blanco para la reforma del gobierno local en España, MAP, 2005

6 Vid. ARENILLA, Manuel, “Principios de la reforma local”, Revista de Estudios Locales, núm.169, 2014.

7 Vid. Sobre la reforma del artículo 135, Vid. RIVERO ORTEGA, Ricardo, Derecho administrativo económico, Marcial Pons, Madrid, 2012. Vid. MERINO ESTRADA, Valentín (Ed.), Crisis económica y entidades locales, Lex Nova, Valladolid, 2012.

9 Vid. CIRCULO CÍVICO DE OPINIÓN, Ocho mil millones de ahorro. La compleja reforma de la Administración local, 7 de mayo de 2013. 
Se comprenden sin embargo las resistencias y críticas al cambio: racionalizar y racionar son verbos distintos, pero apenas se diferencian por cuatro letras. Curiosamente, juntas forman el vocablo "liza", cuyo significado puede ser "campo dispuesto para el combate", o "pelea, lucha". Juego de palabras entretenido para una reforma adversativa, planteada desde su origen lanzando un desafío provocador a los considerados insuficientemente racionales o sostenibles, que debían aceptar el racionamiento.

Confundo deliberadamente racionalizar con racionar porque el adelgazamiento de la Administración local para la estabilidad presupuestaria ha sido el leit motiv de la Ley de Racionalización y Sostenibilidad. Por eso la norma ha resultado tan antipática y ha sido rechazada por todos los grupos políticos en la oposición. Ahí está la explicación de que fuera tan mal recibida por los responsables municipales y autonómicos, perfectamente conscientes del origen del déficit y la deuda en más altos niveles administrativos.

A nadie le gusta que le impongan la dieta, menos aún si le eligen el menú. Y he ahí probablemente una de las causas del rechazo a las previsiones del primer Proyecto de Ley, pues si nadie discutía la necesidad de evitar los desequilibrios presupuestarios, todos preferían disponer de libertad para decidir cómo alcanzar el objetivo. Y debiera respetarse la prioridad de cada cual mientras no se requiera ayuda ajena para conseguirlo. Muchos municipios criticaron que con estas medidas pagaran justos por pecadores.

Y es que la pretendida racionalización podía haberse limitado a las entidades locales con gran deuda (las menos). Además, muchas de las medidas de la nueva Ley fueron anticipadas en acuerdos con las entidades locales, así los reales decretos-leyes de planes de pago a proveedores, medidas que recuerdan a los memorándums de entendimiento (MOUs, en inglés) suscritos por los Estados (España, Grecia, Portugal...) con la Troika, incluyendo las capitulaciones concretas del programa de ayuda financiera.

Personalmente me he manifestado muy crítico con las cesiones de soberanía y autonomía institucional derivadas de estos compromisos con Europa, pero también reconozco que en el caso español el escenario podría haber sido mucho peor. Basta con mirar hacia el oeste y ver el impacto de la crisis sobre las entidades locales portuguesas ${ }^{10}$. E igual que los memorándums de entendimiento han sido mucho más específicos para Portugal que para España, esta reforma local habría contribuido a esquivar otra más drástica, mucho peor.

Ahora bien, ¿Vale conformarse considerando aceptable un mal menor, necesario aunque detestable?, Quienes rechazan por completo la reforma, esgrimen argumentos de inconstitucionalidad variados: lesión de la autonomía local, desnaturalización de la provincia..." ${ }^{11}$. Para muchos expertos, no era esta la reforma apropiada. Pero también habría que preguntarse si en el escenario político y económico en el que se gestó, con las extraordinarias presiones y el ataque especulativo sobre la deuda española en aquel momento, cabía otra alternativa ${ }^{12}$.

\section{OPORTUNIDADES DE LA LEY DE RACIONALIZACIÓN}

Tal vez, ¿hubiera sido mejor dejar todo como estaba aplicando sólo medidas de urgencia y con carácter excepcional? En toda valoración de política pública u opción normativa hemos de incorporar la comparativa de no hacer nada. ¿Hubiera sido suficiente con las Ley de Estabilidad Presupuestaria para corregir cualquier desviación del gasto de las entidades locales? ¿Eran necesarias otras reformas estructurales además de las medidas de excepción? Desde mi punto de vista sí, porque la Administración local debía (y debe) innovarse'3.

A mi juicio, en ningún caso era ni es posible volver a las propuestas de reforma del régimen local consideradas adecuadas en el momento anterior a la crisis económica, cuando los ingresos por recaudación se mantenían estables o crecientes en todos los niveles administrativos. El replanteamiento de la financiación en pleno ajuste hubiera propiciado enfrentamientos poco fructíferos y de resultados aún peores que esta reforma. Así que los condicionantes eran muy distintos en plena crisis, requiriendo otras respuestas ${ }^{14}$.

10 Vid. RIVERO ORTEGA, Ricardo, “Los administrativistas y las crisis", Conclusiones presentadas como relator del Coloquio luso español de profesores de Derecho administrativo celebrado en la Universidad Nova de Lisboa en noviembre de 2013, divulgadas por la AEPDA, 2013.

11 VELASCO CABALLERO, Francisco, "Títulos competenciales y garantía institucional de autonomía local en la Ley de Racionalización y Sostenibilidad de la Administración local”, en SANTAMARÍA PASTOR, Juan Alfonso (Dir.), La reforma de 2013 del Régimen local español, Fundación Democracia y Gobierno local, Barcelona, 2013. ZAFRA VICTOR, Manuel, "Doble inconstitucionalidad del Proyecto de Ley de Racionalización y Sostenibilidad de la Administración local”, en ¿Un nuevo modelo de gobierno local?, Junta de Andalucía, Sevilla, 2014.

12 Vid. FONT I LLOVET, Tomás, “Racionalización y sostenibilidad de la Administración local: ¿Era ésta la reforma?”, Anuario de Gobierno local, núm. 1, 202.

13 Vid. RIVERO ORTEGA, Ricardo, La necesaria innovación en las instituciones administrativas, INAP, Madrid, 2013. MERINO ESTRADA, Valentín/RIVERO ORTEGA, Ricardo, Gestionar mejor, gastar menos. Una guía para la sostenibilidad municipal, Cemci, Granada, 2011.

14 Un ejemplo de reforma local basada en las propuestas anteriores a la crisis lo ofrece la LAULA de Andalucía, Vid. ZAFRA VICTOR, Manuel, “Ley de autonomía local de Andalucía”, Revista de Estudios Regionales, núm. 88, 2010. 
En mi opinión, sin embargo, era y es todavía necesario dar soluciones efectivas para varios problemas acentuados en el escenario presente: discusión sobre la planta; una mejor definición competencial; la garantía de la sostenibilidad de sus servicios básicos; el control del gasto de las entidades locales para su eficiencia; la prevención de la corrupción; la multiplicación exagerada de organizaciones instrumentales dependientes de las entidades locales advertida por el Tribunal de Cuentas ; el crecimiento de plantillas en períodos preelectorales... ${ }^{15}$

La Ley de Racionalización y Sostenibilidad ha tenido la oportunidad de resolver varias de estas necesidades. $\mathrm{Y}$ en honor a la verdad debe decirse que lo ha intentado en parte, aunque muy presionada y condicionada por el objetivo de traducir en resultados económicos sus logros y alcances. Incorporar ese elemento de restricción presupuestaria a debates tan sensibles como el relativo al papel de los pequeños municipios, las entidades locales menores o las competencias sociales de los ayuntamientos, explica la airada reacción municipalista ${ }^{16}$.

Paradójicamente el principal objetivo de la Ley, cual es propiciar la contención de gasto, se alcanzó antes y por otras vías. Es lo que se ha definido perspicazmente como efecto anticipado de la reforma, aunque como ya hemos insinuado cabría preguntarse si se hubiera producido el mismo efecto sin esta Ley; si hubiera sido suficiente con la Ley de Economía Sostenible. Mi respuesta es sencilla: en el corto plazo, hubiera bastado con las medidas de estabilidad; en el medio y largo plazo, era imprescindible la reforma local'17.

$Y$ es que las limitaciones y controles del gasto previstos en la Ley Orgánica de Estabilidad Presupuestaria son eminentemente cuantitativos, mientras el Gobierno y la Administración local requerían a mi juicio indicadores cualitativos. Establecer prioridades en cuanto a las competencias y servicios a garantizar (los más básicos antes que otros menos imprescindibles), señalar topes máximos sobre el coste del personal político y eventual, o exigencias precisas sobre la excesiva proliferación de entidades instrumentales ${ }^{18}$.

Para todo ello también era necesario reforzar el papel de los interventores, lo que relativamente parece intentarse con un nuevo régimen de los habilitados, así como con la modificación del Texto Refundido de la Ley de Haciendas locales, en su artículo 213, contemplando el envío de información de los órganos interventores al Ministerio de Hacienda. O en su artículo 218, previendo un Informe sobre resolución de discrepancias, que será discutido en el Pleno. También se prevé la comunicación de las irregularidades detectadas al Tribunal de Cuentas.

Además, la Disposición Adicional Sexta del Proyecto de Ley permite articular la colaboración con la Intervención General del Estado de las intervenciones locales. Pero si no se refuerza el estatuto de los habilitados de carácter nacional despejando dudas relevantes sobre su papel a la hora de señalar ilegalidades (el complejo debate sobre la llamada "advertencia de ilegalidad") o se establecen límites a los nombramientos por libre designación, de poco servirán todas estas novedades ${ }^{19}$.

En muchos de los puntos más relevantes del cambio normativo, la Ley de Racionalización y Sostenibilidad opta por soluciones comedidas, de equilibrio o composición de posturas, evitando las alternativas radicales y huyendo de la extrema satisfacción de ciertas demandas. Así ocurre en lo relativo a los habilitados (pues la posición de la FEMP no coincidía con la de COSITAL), pero también en otros asuntos mayores, como el debate sobre el excesivo número de entidades locales en España.

Muy criticado por tirios y troyanos ha sido el posicionamiento de la Ley de Racionalización y Sostenibilidad sobre la espinosa cuestión de la planta municipal. Partimos de un mapa local apenas alterado desde la Edad Media, realidad perceptible a simple vista de proyección de los términos municipales sobre el perfil de la península. El inframunicipalismo es más acusado cuanto más al norte, multiplicándose también por cientos las entidades locales menores que gestionan sus bienes y autogobiernan sus comunidades ${ }^{20}$.

La nueva regulación de las entidades locales menores, recogida en el artículo 24bis de la Ley, más los incentivos económicos previstos para la fusión de municipios, son buena muestra de la apuesta del legislador por la voluntariedad, reflejada en otros momentos clave de la norma (así, por ejemplo, en lo relativo a la función coordinadora de las diputaciones provinciales). No se elude la necesidad de racionalizar también reduciendo cifras, aunque es muy remota en mi opinión la posibilidad de que se multipliquen las fusiones.

15 Vid. PARADA VÁZQUEZ, José Ramòn/FUENTETAJA PASTOR, Jesús, Reforma y retos de la Administración local, Marcial Pons, 2007.

16 Se convocaron manifestaciones y protestas en toda España contra la reforma local, señalando su potencial impacto negativo sobre los servicios sociales y la existencia misma de los pequeños municipios, probablemente con un alarmismo que la realidad ha demostrado exagerado, a la vista de los hechos.

17 Vid. RIVERO ORTEGA, Ricardo, “¿Cómo hacer sostenible la iniciativa económica local?”, Revista de Estudios Locales, 2011.

18 Vid. RIVERO ORTEGA, Ricardo, “Limitaciones a la potestad de autoorganización. Las formas de prestación de los servicios locales”, Diputación de Barcelona, 2013.

19 Al respecto me remito a las ponencias y conclusiones alcanzadas en el último Congreso de COSITAL, celebrado en el INAP en noviembre de 2014.

20 Vid. DÍEZ SÁNCHEZ, Juan José (Ed.), La planta del gobierno municipal, AEPDA, 2013. 
No creo que la nueva redacción del artículo 13 de la Ley de Bases favorezca realmente la reducción del número de municipios porque obvia dos aspectos muy sensibles y explicativos e la vocación identitaria de las colectividades locales, ambos basados en el interés: la administración de los bienes comunales, por un lado, y los actos de adjudicación de contratos (selección de proveedores y de personal), por otro. Deslocalizar esas decisiones de reparto de beneficios suscita un sinfín de resistencias, y no sólo en clave sentimental.

Menos componedora y transigente se muestra en cambio la reforma en lo relativo a la exigencia de racionalización ad intra de las estructuras municipales, de modo y manera que es previsible un mayor impacto organizativo de sus previsiones sobre la Administración instrumental, tanto sobre el número de entidades públicas empresariales y sociedades mercantiles, como sobre los consorcios. Y no sólo respecto de las creadas en el pasado, sino también hacia el futuro (nueva redacción del artículo 84bis) ${ }^{21}$.

Iguales efectos reales puede producir la reforma sobre el gasto en personal, conteniendo las tendencias retributivas descompensadas que por la vía del reconocimiento de complementos específicos se observan en la Administración local. El nuevo artículo 75bis, además, establece topes para los salarios de los cargos políticos, límites muy generosos y elevados que sólo servirán para corregir las situaciones más exageradas en municipios que retribuían muy por encima de la media a sus ediles ${ }^{22}$.

Estas medidas también han de aplicarse en las sociedades mercantiles, regulando con bastante detalle tanto el número máximo de miembros en los órganos de gobierno en la nueva redacción de la Disposición adicional duodécima de la Ley de bases. Igualmente se contempla la transparencia necesaria de estos datos y la extinción de los contratos mercantiles de alta dirección, como se establecen topes de sueldos y limitaciones del personal eventual en la Disposición Transitoria Octava y Disposición Transitoria Undécima²3.

Todos estos cambios en línea de austeridad y buen gobierno son, sin embargo, menores en comparación con el tema estrella de la reforma local, cual es el nuevo esquema competencial. En la reordenación de las competencias municipales y provinciales se encuentra una de las modificaciones más relevantes de la normativa básica de régimen local, tratando de eliminar duplicidades y solapamientos para realizar por fin el principio "una Administración, una competencia" (mantra del informe CORA) ${ }^{24}$.

Es fundamental a estos efectos el nuevo apartado 4 del artículo 7: “Las entidades locales sólo podrán ejercer competencias distintas de las propias y de las atribuidas por delegación cuando no se ponga en riesgo la sostenibilidad financiera del conjunto de la Hacienda municipal, de acuerdo con los requerimientos de la legislación de estabilidad presupuestaria y sostenibilidad financiera y no se incurra en un supuesto de ejecución simultánea del mismo servicio público con otra Administración Pública. A estos efectos, serán necesarios y vinculantes los informes previos de la Administración competente por razón de materia, en el que se señale la inexistencia de duplicidades, y de la Administración que tenga atribuida la tutela financiera sobre la sostenibilidad financiera de las nuevas competencias. En todo caso, el ejercicio de estas competencias deberá realizarse en los términos previstos en la legislación del Estado y de las Comunidades Autónomas".

Así, el nuevo régimen de las llamadas competencias impropias requiere una reconsideración en clave de coordinación interadministrativa y justificación de sostenibilidad de su prestación. Comunidades autónomas y entidades locales habrían de alcanzar acuerdos en materias principales (educación, sanidad, servicios sociales) evitando los solapamientos y clarificando las fuentes de financiación de tales intervenciones, una vez más con el propósito de garantizar una Administración eficiente desde el punto de vista económicos ${ }^{25}$.

Muchas objeciones ha recibido tanto en su planteamiento teórico como en sus consecuencias prácticas esta exigencia: ¿Acaso no pueden ser positivas determinadas duplicidades?; ¿Quién se solapa y duplica?; ¿No pueden dos administraciones prestar el mismo servicio ante una demanda múltiple y diversa? En todo caso, en atención al principio de subsidiariedad, de ser preciso eliminar intervenciones en niveles administrativos, habría que comenzar por los más distantes, reconsiderando estructuras estatales o autonómicas ${ }^{26}$

21 Vid. NIETO GARRID, Eva, “La Ley de racionalización y sostenibilidad de la Administración local y los consorcios administrativos”, en CARRILLO DONAIRE, Juan Antonio/NAVARRO RODRÍGUEZ, Pilar (Dirs.), La reforma del Régimen Jurídico de la Administración local, 2014.

22 Vid. RIVERO ORTEGA, Ricardof/MERINO ESTRADA, Valentín, "Benchmarking, ejemplaridad pública y Nudge: herramientas de buen gobierno, integridad institucional y la innovación democrática”, Revista Fundación Democracia y Gobierno Local, 2012.

23 Vid. FERNÁNDEZ RAMOS, Severiano, “Los falsos empleados públicos: el personal eventual”, Revista General de Derecho Administrativo, núm. 34, 2013.

24 Vid. CALVO, Juan, “La nueva delimitación competencial y organizativa de la Administración local”, en Revista de Estudios Locales, núm. 169, 2014.

25 Vid. MORILLO-VELARDE, José Ignacio, “Competencias municipales: competencias delegadas e impropias”, Cuadernos de Derecho local, núm.34, 2014.

26 Vid. MERINO ESTRADA, Valentín, “Los servicios públicos locales tras la reforma”, En Revista de Estudios Locales, núm. $169,2014$. 
Para ofrecer un balance justo y realista de la reforma, hay que esperar a tener más datos en torno a su incidencia final sobre educación, sanidad y servicios sociales. Las decisiones autonómicas en línea de relativizar las exigencias de la nueva Ley atemperarán sus consecuencias, pero sería interesante disponer de datos concretos sobre competencias impropias abandonadas a resultas de la suma normativa y económica en el contexto de la crisis: ¿Puede hablarse de un retroceso de la intervención social local?

Probablemente la respuesta será distinta dependiendo de la capacidad económica real de cada Ayuntamiento, porque las cifras importan en esto como en todo. Del mismo modo, la reforma competencial no afecta de igual forma a los grandes ayuntamientos y a los medianos y pequeños. A los primeros intenta apartarlos de algunos ámbitos de acción tradicionales, pero a los de menos de 20.000 habitantes, que son la inmensa mayoría, simplemente parece pretender sustraerles sus servicios básicos para encomendárselos a las diputaciones, convertidas en responsables de su prestación en condiciones eficientes ${ }^{27}$.

Esta apuesta clara por las diputaciones provinciales ha suscitado todo tipo de críticas e incluso un pronunciamiento del Consejo de Estado que cuestiona la claridad de la redacción normativa en el punto de su papel coordinador de la prestación de servicios. La realidad además está mostrando un escaso interés de los gobiernos provinciales por cambiar su papel tradicional, pues muchas de sus líneas demuestran el caso omiso prestado a la eliminación de la genérica competencia de fomento provincial.

De poco sirve que el legislador se empeña en convertir a las diputaciones provinciales en los nuevos actores administrativos de la ordenación del territorio si los poderes autonómicos tienen planes alternativos en este esquema. Escasos resultados dará el replanteamiento competencial si todos los entes locales siguen actuando como antes. Mínimas reducciones del número de municipios veremos sin enfoques más pragmáticos. ¿Se aprovecharán algún día las oportunidades de la reforma local? Contesto prudentemente sí, pero también pienso que se podía hacer avanzando mucho más en clave de innovación ${ }^{28}$.

\section{RIESGOS DE LA LEY DE RACIONALIZACIÓN Y SOSTENIBILIDAD}

El balance final de las oportunidades creadas y aprovechadas por la reforma legal apenas compensa tanto alarmismo y resonancia acusándola de atentado al municipalismo. Efectivamente, si la Ley de Racionalización y Sostenibilidad me parece necesaria, también podría haber incorporado soluciones más cautas sobre servicios sensibles y esenciales en el momento de crisis que aún atravesamos. Esto es predicable, por ejemplo, de las escuelas infantiles o las residencias de la tercera edad.

¿Ha puesto en riesgo la Ley servicios esenciales no considerados prioritarios? Probablemente no, pero sí ha propiciado la confusión sobre su mantenimiento de futuro, lo cual es negativo. Y este es un efecto paradójico de la reforma, porque probablemente la intención del legislador era garantizar la sostenibilidad de estos servicios al exigir un comportamiento presupuestario más responsable de los gestores locales, exigiéndoles también la fijación de un orden de prioridades.

Algo similar ha sucedido - o sucederá pronto - con el refuerzo del protagonismo de las diputaciones provinciales, incapaces hasta el momento de asumir ese papel que pretende concedérsele. Su pasividad en la aplicación de las previsiones de la reforma ha reabierto el debate sobre la procedencia de su supresión, con voces a favor y en contra, en una polémica últimamente protagonizada por José Luis RIVERO YSERN y Francisco SOSA WAGNER, dos señalados expertos en Derecho local ${ }^{29}$

Parece muy necesaria la reinvención de las diputaciones, pero éstas apenas han adoptado estrategias adaptativas al nuevo contexto. En cambio, están liderando iniciativas de promoción del empleo y el desarrollo económico que no han sido cuestionadas en términos competenciales, aunque si no se articulan en coordinación con las competencias autonómicas o municipales carecen de habilitación expresa en la nueva enumeración de títulos del artículo 36 de la Ley de Bases de Régimen local.

En suma, la oportunidad de reinvención de las diputaciones ha sido servida por la Ley, pero lo cierto es que hasta ahora no parece haber sido aprovechada por los responsables de los poderes provinciales, más interesados al parecer en gestionar como siempre que en explorar las posibilidades de mejora de la gestión de los servicios de los pequeños municipios. Una vez se superen los peores escenarios económicos de la crisis, se hará mucho más difícil el liderazgo provincial en sus funciones de coordinación.

27 Vid. RIVERO ORTEGA, Ricardo, “Alteraciones de la planta municipal y reinvención de las diputaciones”, en Revista de Estudios Locales, núm. 169, 2014.

28 Vid. MERINO ESTRADA, Valentín/RIVERO ORTEGA, Ricardo, Innovación en los gobiernos locales, INAP, Madrid, 2014.

29 Vid. RIVERO YSERN, José Luis, “La Provincia y la Ley de Racionalización y Sosteniblidad de la Administración local” Revista General de Derecho administrativo, núm. 36, 2014. SOSA WAGNER, Francisco, “El cangilón de la provincia”, Diario de Derecho municipal, lustel, 2014. 
De persistir esta inercia institucional, habría que plantearse en futuras reformas constitucionales la disponibilidad de la provincia como entidad local, generalizando un diseño asimétrico que funciona de hecho para las Comunidades autónomas uniprovinciales, y podría ser extensible a otras con menos apego a las diputaciones. Así, cada Estatuto de Autonomía podría definir - previa reforma constitucional, insisto - si deben existir también poderes locales provinciales u otras fórmulas alternativas.

De este modo se superaría el escenario resultante de la interpretación constitucional plasmada de modo traumático en la Sentencia del Tribunal Constitucional 31/2010, de 28 de junio, recaída sobre el Estatuto de Autonomía de Cataluña ${ }^{30}$. Y se daría salida al más que creciente interés de las Comunidades Autónomas sobre el régimen local, obstáculo principal a la efectividad de las previsiones de la Ley de Racionalización y Sostenibilidad, como advirtió certeramente Santiago MUÑOZ MACHADO31.

\section{LA DESACTIVACIÓN DE LA LEY POR LOS PODERES AUTONÓMICOS}

La Ley de Racionalización y Sostenibilidad de la Administración local reconoce en sus propias disposiciones la asimetría del régimen local en el territorio español. Así, la Disposición Adicional primera prevé el régimen aplicable a la Comunidad Autónoma del País Vasco; la Disposición Adicional segunda, el aplicable a la Comunidad Foral de Navarra; la Disposición adicional tercera contiene una mención específica para Aragón, en su apartado tercero; la Disposición Adicional cuarta contempla especialidades para Ceuta y Melilla... ${ }^{32}$

Las resistencias de los poderes autonómicos al incremento del protagonismo del legislador básico sobre el régimen local se comprenden tras una oleada de reformas estatutarias cuyo propósito incluyó el avance de la “interiorización” de esta normativa, en una línea federal defendida por muchos de nuestros expertos, pero no necesariamente la única posible ni la más plausible a la vista del texto constitucional, máxime si tenemos presentes las competencias estatales del artículo 149.1.1833.

No es casual que hayan sido precisamente las Comunidades Autónomas con versiones más recientes de sus Estatutos de Autonomía las más resistentes y activas en la defensa de sus propias competencias contra las medidas incorporadas en la Ley de Racionalización. La previsión en muchas de ellas de la existencia de leyes locales autonómicas se ha visto seriamente afectada por un texto estatal decidido a poner orden en un mundo a ordenar territorialmente, materia propia del poder autonómico ${ }^{34}$.

La aprobación en Andalucía de la LAULA (Ley de Autonomía Local de Andalucía) es un buen ejemplo de los problemas de fuentes generados por la aprobación final de la Ley de racionalización. Algunas de las previsiones de este texto ya parecían incompatibles con otras de la Ley de Bases, pero la última redacción del Estatuto de Autonomía andaluz podría explicar desavenencias en aspectos clave (como los modos de gestión de los servicios públicos). La reforma ha incrementado estas contradicciones ${ }^{35}$.

No es en modo sólo una cuestión política, pues entre los contradictores de la Ley puede señalarse a la Comunidad Autónoma de Castilla y León, emblemática por su número elevadísimo de municipios y por tramitar en paralelo a la reforma del Estado una iniciativa alternativa - no contraria, pero difícilmente complementaria - en forma de Ley de Ordenación, Servicios y Gobierno del Territorio, norma que apuesta por fórmulas alternativas a la preferida por el Estado (las diputaciones) para mejorar la gestión de los servicios municipales ${ }^{36}$.

Así, en Castilla y León se aprobó el Decreto-Ley 1/2014, de 27 de marzo, de medidas urgentes para la garantía y continuidad de los servicios públicos en Castilla y León, derivado de la entrada en vigor de la Ley 27/2013, de 27 de diciembre, de racionalización y sostenibilidad de la Administración local. Medidas similares encontramos en Cataluña, Aragón, Andalucía o en las Islas Baleares (el Decreto Ley 2/2014, de 21 de noviembre, de medidas urgentes para la aplicación de la Ley 27/2013, 27 de diciembre, de racionalización y sostenibilidad de la Administración local).

Las Comunidades Autónomas han satisfecho su prurito de defensa de competencias propias e impropias ajenas con estas medidas de urgencia, pero cabría preguntarse si realmente se blindan así las competencias loca-

30 Vid. FERNÁNDEZ MONTALVO, Rafael, “Los gobiernos locales intermedios en el Estado autonómico después de la Sentencia del Tribunal Constitucional 31/2010, de 28 de junio”, Cuadernos de Derecho Público, núm. 37.

31 Vid. MUÑOZ MACHADO, Santiago, en la posición del Círculo Cívico de Opinión sobre la reforma.

32 Vid. VELASCO CABALLERO, Francisco, “Aplicación asimétrica de la Ley de Racionalización y Sostenibilidad de la Administración local”, Anuario de Derecho Municipal, núm. 7, 2013.

33 Vid. Las posiciones respectivas sobre la autonomía local defendidas por José Luis CARRO FERNÁNDEZ-VALMAYOR y Francisco VELASCO CABALLERO en el Congreso de la AEPDA celebrado en Santander, publicadas en 2007.

34 Vid. RIVERO ORTEGA, Ricardo/MERINO ESTRADA, Valentín (Dirs.), Derecho local de Castilla y León, lustel, 2014.

35 Vid. RIVERO ORTEGA, Ricardo, “régimen jurídico de los servicios públicos locales”, en Tratado de gestión de los servicios públicos, La Ley, 2012.

36 Vid. Derecho local de Castilla y León, cit, lustel, 2014. 
les ${ }^{37}$. La protección de los ámbitos de intervención de municipios y diputaciones provinciales requiere, desde mi punto de vista, más participación de las entidades locales sobre los tributos autonómicos, pues las normas por sí solas no garantizan la viabilidad económica.

\section{PERSPECTIVAS DE FUTURO}

Con esta reacción de parte de las Comunidades Autónomas, en cualquier caso, se dificultan muchas de las consecuencias prácticas de la reforma, pudiendo formularse las siguiente preguntas como indicador de su aplicación real: ¿Están realizándose los informes sobre competencias previstos en del 3.2.4 de la Ley? ; ¿Están preparándose las diputaciones provinciales para asumir su nuevo papel coordinador?; ¿Se han llevado a cabo fusiones gracias a los incentivos prometidos?; ¿Cuántas entidades instrumentales se han suprimido realmente?

Las opiniones más alarmistas en relación a las consecuencias de esta Ley no parecen haberse visto correspondidas por la realidad de los hechos, porque la norma no ha sido redactada ni aplicada en versiones maximalistas. No se ha producido una reducción drástica ni de estructuras ni de competencias, aunque sí es muy probable que en adelante las entidades locales calibren más en términos de sostenibilidad la creación de nuevos órganos o la prestación de más servicios, ponderando sus costes.

El Auto del Tribunal Constitucional de 23 de septiembre de 2014 deniega la petición de suspensión de vigencia de la Ley, solicitada por el Parlamento de Cataluña en relación a varias de sus disposiciones. El dictamen emitido por el Consejo de Estado señala alguna posible tacha de inconstitucionalidad, pero en todo caso salvable por la vía de la interpretación conforme. Ya se ha aprobado la normativa de desarrollo del cálculo de los costes efectivos de los servicios, mediante el dictado de la Orden de 6 de noviembre de 2014.

La Ley, por tanto, sigue plenamente vigente, demostrando sus inefectividades que para las grandes reformas es preferible el consenso a la unilateralidad. Que cuando no se plantean en términos positivos, sino bajo la presión de una extrema urgencia, no persisten en el tiempo. Que en España los municipios son anteriores al Estado, a las Comunidades Autónomas y a las Diputaciones provinciales. Que la autonomía es fuerte y resistente por sus bases democráticas. Que no es nada fácil "racionalizar" la Administración local.

37 Vid. NAVARRO RODRÍGUEZ, Pilar/ZAFRA VICTOR, Manuel, “El pretendido blindaje autonómico de las competencias municipales tras la reforma de la Administración local”, Revista de Estudios de la Administración local y autonómica, núm. $2,2014$. 